\title{
Skew products and minimal dynamical systems on separable Hilbert manifolds
}

\author{
A. FATHI \\ G.R. 21 du CNRS, Université Paris-Sud, Bâtiment 425, F-91405 Orsay cedex, France
}

(Received 14 March 1983)

Abstract. We prove that any locally compact, non-compact, second countable group acts minimally on any metrizable connected manifold modelled on the separable Hilbert space.

\section{Introduction}

The purpose of this article is to prove the following theorem.

THEOREM. If $M$ is a connected separable Hilbert manifold and $G$ is a locally compact, non compac, second countable topological group, we can find a continuous and minimal action of $G$ on $M$.

This theorem is a generalization of the one we obtained in [5]. Let us point out that, as in [5], we do not construct smooth actions in the case where $G$ is a Lie group. To our knowledge, it is still not known that there exists a minimal diffeomorphism of $l^{2}$.

The main ingredients for this paper are our previous work [5] and the paper of Glasner and Weiss [6] about existence of minimal skew product extension. In fact, it is quite natural, once one knows the existence of a minimal action on Hilbert space, to try to apply the ideas of Glasner and Weiss and to lift this action on $l^{2}$ to a minimal skew product extension on $l^{2} \times M$ which is homeomorphic to $M$. Unfortunately, this is impossible due to the non-compactness of $M$. However it is easy to construct skew product extensions which have dense orbits, and as we showed in [5] it is pretty natural in the context of infinite dimensional topology to try to construct extensions such that the subset of points of $l^{2} \times M$ with a dense orbit is homeomorphic to $l^{2} \times M$.

It is not necessary to be familiar with [5] or [6] to read this paper. In fact we obtain, as a by product, a generalization of the theorem of Glasner and Weiss to a general group and a general base space (see theorem 4.3).

\section{Topology on the space of skew products}

We consider a topological space $X$ endowed with a continuous action $\alpha$ of a locally compact group $G$. Since this action is fixed in the sequel we will denote the effect of $g \in G$ on $x \in X$ by $g x$ instead of $\alpha(g, x)$. We suppose that $Y$ is a metric space, whose metric is denoted by $d$. Without loss of generality, we can assume that the metric $d$ is bounded by 1 (replace $d(x, y)$ by $\min [1, d(x, y)]$ ). 
We will consider actions $A$ of $G$ on $X \times Y$ which are skew products over $\alpha$. Such an action $A: G \times X \times Y \rightarrow X \times Y$ can be written as $A(g, x, y)=\left(g x, a_{g, x}(y)\right)$. We will call $a_{g, x}$ the cocycle associated to $A$. It verifies the cocycle equations:

$$
\begin{aligned}
a_{g^{\prime}, g x} \circ a_{g, x} & =a_{g^{\prime} g, x}, & & \forall x \in X, \forall g \in G, \forall g^{\prime} \in G, \\
a_{e, x} & =\operatorname{Id}_{Y}, & & \forall x \in X,
\end{aligned}
$$

( $e$ is the neutral element in $G$ ).

We denote by $\mathscr{S}(\alpha)$ the set of skew products on $X \times Y$ over $\alpha$. For each compact subset $C$ of $G$, we define a semi-metric $D_{C}$ on $\mathscr{S}(\alpha)$ by:

$$
D_{C}\left(A, A^{\prime}\right)=\sup \left\{d\left(a_{g, x}(y), a_{g, x}^{\prime}(y)\right) \mid g \in C, x \in X, y \in Y\right\},
$$

where $\left(a_{g, x}\right)$ and $\left(a_{g, x}^{\prime}\right)$ are the cocycles associated with $A$ and $A^{\prime}$.

The set of semi-metrics $\left\{D_{C} \mid C\right.$ a compact subset of $\left.G\right\}$ defines a uniform structure on $\mathscr{S}(\alpha)$ which is easily seen to be Hausdorff. Moreover if $G$ is $\sigma$-compact, it is easy to define a metric on $\mathscr{S}(\alpha)$ which gives the same uniform structure.

LEMMA 1.1. If $Y$ is complete for $d$, then $\mathscr{S}(\alpha)$ is complete. In particular if $G$ is $\sigma$-compact, $\mathscr{S}(\alpha)$ is metric complete.

Proof. Suppose $\left(A^{i}\right)_{i \in I}$ is a Cauchy net in $\mathscr{S}(\alpha)$ with associated cocycles $\left(a_{\mathrm{g}, x}^{i}\right)$. It is easy to show that there exists a continuous map $G \times X \times Y \rightarrow Y,(g, x, y) \mapsto a_{g, x}(y)$, such that if we put $A(g, x, y)=\left(g x, a_{g, x}(y)\right)$, we have, for each compact subset $C$ of $G, D_{C}\left(A^{i}, A\right) \rightarrow 0$ as $i$ goes to infinity in $I$. We still must check that $A$ is an action of $G$ on $X \times Y$. This means that we have to prove that $a_{g, x}$ verifies the cocycle conditions.

Suppose $g, g^{\prime} \in G, x \in X$ and $y \in Y$; we have for each $i \in I$ :

$$
\begin{aligned}
d\left[a_{g^{\prime}, g x}\left(a_{g, x}(y)\right), a_{g^{\prime}, x}(y)\right] \leq & d\left[a_{g^{\prime}, g x}\left(a_{g, x}(y)\right), a_{g^{\prime}, g x}\left(a_{g, x}^{i}(y)\right)\right] \\
& +d\left[a_{g^{\prime}, g x}\left(a_{g, x}^{i}(y)\right), a_{g^{\prime}, g x}^{i}\left(a_{g, x}^{i}(y)\right)\right] \\
& +d\left[a_{g^{\prime}, g x}^{i}\left(a_{g, x}^{i}(y)\right), a_{g^{\prime}, x}^{i}(y)\right]+d\left[a_{g^{\prime}, x}^{i}(y), a_{g^{\prime}, x}(y)\right] .
\end{aligned}
$$

Now, as $i$ goes to infinity, it is clear that the second and fourth terms go to zero because they are bounded respectively by $D_{\left\{g^{\prime}\right\}}\left(A, A^{i}\right)$ and $D_{\left\{g^{\prime} g\right\}}\left(A, A^{i}\right)$. The first term goes to zero as $i$ goes to infinity because $a_{g, x}^{i}(y) \rightarrow a_{g, x}(y)$ and $a_{g^{\prime}, g x}$ is a continuous map. The third term is zero because of the cocycle conditions on $\left(a_{g, x}^{i}\right)$. Of course we have $a_{e, x}=\operatorname{Id}_{Y}$, for all $x \in X$, because this is true for each $a_{e, x}^{i}$.

LEMMA 1.2. The topology defined on $\mathscr{S}(\alpha)$ is finer than the compact open topology.

Proof. Let $p_{1}$ and $p_{2}$ be the canonical projections of $X \times Y$ on the two factors. The topology on $\mathscr{P}(\alpha)$ is the topology of uniform convergence of the maps $p_{2} \circ \mathrm{A}$ : $G \times X \times Y \rightarrow Y$ on each set of the form $C \times X \times Y$, where $C$ is an arbitrary compact subset of $G$. Certainly this topology is finer than the compact open topology on the maps from $G \times X \times Y$ to $Y$.

This proves the lemma since $p_{1} \circ A$ is independent of $A \in \mathscr{S}(\alpha)$.

We now introduce a subset $\tilde{\mathscr{S}}\left(\operatorname{Id}_{X}\right)$ of $\mathscr{P}\left(\operatorname{Id}_{X}\right)$ the set of skew products on $X \times Y$ over the identity of $X$. The set $\tilde{\mathscr{F}}\left(\operatorname{Id}_{X}\right)$ is defined as the set of homeomorphisms $H$ of $X \times Y$ of the form $H(x, y)=\left(x, h_{x}(y)\right)$ such that the two sets $\left(h_{x}\right)_{x \in X},\left(h_{x}^{-1}\right)_{x \in X}$ 
of self maps of $Y$ are uniformly equicontinuous (with respect to $d$ ). It is easy to verify that $\tilde{\mathscr{S}}\left(\mathrm{Id}_{X}\right)$ is a group (no topology involved here).

Given $A$ in $\mathscr{S}(\alpha)$ and $H$ in $\tilde{\mathscr{S}}\left(\operatorname{Id}_{X}\right)$, we can define $H A H^{-1}$ by

$$
H A H^{-1}(g, x, y)=H\left(A\left(g, H^{-1}(x, y)\right)\right) \text {. }
$$

It is clear that $H A H^{-1}$ is in $\mathscr{S}(\alpha)$.

LEMMA 1.3. If $H \in \tilde{\mathscr{S}}\left(\operatorname{Id}_{X}\right)$, the map $\mathscr{S}(\alpha) \rightarrow \mathscr{P}(\alpha), A \rightarrow H A H^{-1}$ is a homeomorphism. Proof. We have only to check that it is continuous. Since $H$ is in $\tilde{\mathscr{S}}\left(\operatorname{Id}_{X}\right)$, the function $\theta: \mathbb{R}_{+} \rightarrow \mathbb{R}_{+}$defined by

$$
\theta(\delta)=\sup \left\{d\left(h_{x}(y), h_{x}\left(y^{\prime}\right)\right) \mid x \in X, y, y^{\prime} \in Y, \quad d\left(y, y^{\prime}\right) \leq \delta\right\}
$$

satisfies $\lim _{\delta \rightarrow 0} \theta(\delta)=0$. The lemma follows then from the easily verified inequality:

$$
D_{C}\left(H A H^{-1}, H A^{\prime} H^{-1}\right) \leq \theta\left(D_{C}\left(A, A^{\prime}\right)\right) \text {. }
$$

\section{Construction of some real valued functions}

Let $G$ be a locally compact group, $C$ a compact subset of $G$ and $n$ a positive integer. We define a function $\varphi_{n, C}: G \rightarrow[0,1]$ by:

$$
\begin{gathered}
\varphi_{n, C}(C)=1 ; \\
\varphi_{n, C}\left(C^{m+1}-C^{m}\right)=\max ((n-m) / n, 0), \quad \forall m \in \mathbb{N}^{*} ; \\
\varphi_{n, C}=0 \quad \text { outside } \bigcup_{m \in N^{*}} C^{m} .
\end{gathered}
$$

We state some properties of $\varphi_{n, C}$ in the following lemma.

LEMMA 2.1. $\varphi_{n, C}$ is (Borel) measurable; $\varphi_{n, C}(C)=1, \varphi_{n, C}(G) \subset[0,1] ; \varphi_{n, C}=0$ outside $\bigcup_{i=1}^{n} C^{i}$. Moreover if $C$ is symmetric (i.e. $C=C^{-1}=\left\{g^{-1} \mid g \in C\right\}$ ), we have for each $\mathrm{g} \in C$ and $\mathrm{g}^{\prime} \in \mathrm{G}$;

$$
\left|\varphi\left(g^{\prime} g\right)-\varphi\left(g^{\prime}\right)\right| \leq \frac{1}{n} \quad \text { and }\left|\varphi\left(g g^{\prime}\right)-\varphi\left(g^{\prime}\right)\right| \leq \frac{1}{n} .
$$

Proof. The first four properties are trivial. The last one follows from the fact that if $C$ is symmetric, we have: $g \in C, g^{\prime} \in C^{m+1} \backslash C^{m}$ implies $g g^{\prime}$ and $g^{\prime} g$ belong to

$$
C^{m+2} \backslash C^{m-1} \subset C^{m+2} \backslash C^{m+1} \cup C^{m+1} \backslash C^{m} \cup C^{m} \backslash C^{m-1} .
$$

We will need the following well-known technical lemma. We will provide the proof, since it is very short.

LEMMA 2.2. Suppose $\alpha: G \times X \rightarrow X$ is a continuous action of the group $G$ on $X$. If $K \subset G$ is compact and $A \subset X$ is closed, then the set $\alpha(K \times A)$ is closed in $X$.

Proof. Since $\alpha$ can be written as the composition of the homeomorphism $G \times X \rightarrow$ $G \times X,(g, x) \mapsto(g, \alpha(g, x))$ followed by the projection $G \times X \rightarrow X$, it suffices to prove that if $K$ is a compact space, the projection $p: K \times X \rightarrow X$ is closed. Let $B$ be a closed subset of $K \times X$, we have:

$$
X \backslash p(B)=\{x \in X \mid K \times\{x\} \subset K \times X \backslash B\} .
$$

Using the compactness of $K$, it is very easy to show that this last set is open in $X$. 
We can now state and prove the main lemma of this work.

LEMMA 2.3. For each compact subset $C$ of $G$ and each $\varepsilon>0$, we can find a compact subset $C^{\prime}$ of $G$ which satisfies the following property:

Given a continuous action $\alpha$ of $G$ on a normal space $X$, a closed subset $F$ of $X$, with $g F \cap F=\varnothing$ for all $g \in G, g \neq e$, and a neighbourhood $V$ of $C^{\prime} F=\left\{g x \mid g \in C^{\prime}, x \in F\right\}$, there exists a continuous map $\theta: X \rightarrow[0,1]$ such that:

(i) $\theta(F)=1$;

(ii) $\theta=0$ outside $V$;

(iii) $\forall g \in C, \forall x \in X,|\theta(g x)-\theta(x)|<\varepsilon$.

Proof. We can suppose that $C$ is a symmetric neighbourhood of $e$ in $G$. Let $\mu$ be a right invariant Haar measure on $G$. Choose $C_{0}$ a compact symmetric neighbourhood of $e$ such that $C_{0}^{2} \subset \dot{C}$, the interior of $C$. We have $\mu\left(C_{0}\right)>0$. Fix $n$ such that $2 \mu(C) / n \mu\left(C_{0}\right)<\varepsilon$. Put $C^{\prime}=C^{n+1}$. Suppose now $\alpha, X, F$ and $V$ are given as stated above. Using the inclusion $C_{0}^{2} \subset \dot{C}$ and the fact that $g F \cap F=\varnothing$, for all $g \neq e$, we have

$$
\left[\left(C^{2 n}-\dot{C}\right) C_{0} F\right] \cap C_{0} F=\varnothing .
$$

Since our space $X$ is normal, we can separate the two closed (see lemma 2.2) sets $\left(C^{2 n}-\stackrel{\circ}{C}\right) C_{0} F$ and $C_{0} F$ by two open sets $W$ and $W^{\prime}$. Using the compactness of $\left(C^{2 n}-\dot{C}\right)$ and $C^{n}$ we can find an open neighbourhood $U$ of $C_{0} F$ such that $U \subset W^{\prime}$, $\left(C^{2 n}-\stackrel{\circ}{C}\right) U \subset W, C^{n} U \subset V$ (recall that $V$ is a neighbourhood of $C^{n+1} F=C^{\prime} F$ ). So up to this point we have found a neighbourhood $U$ of the closed set $C_{0} F$ such that:

$$
\left[\left(C^{2 n}-\stackrel{\circ}{C}\right) U\right] \cap U=\varnothing \text { and } C^{n} U \subset V .
$$

We now choose a continuous map $\rho: X \rightarrow[0,1]$ such that $\rho\left(C_{0} F\right)=1, \rho=0$ outside $U$. We consider the map $\varphi_{n, C}$ defined above and we define $\theta_{1}: X \rightarrow[0, \infty[$ by:

$$
\theta_{1}(x)=\int_{G} \varphi_{n, C}(k) \rho(k x) d \mu(k)=\int_{C^{n}} \varphi_{n, C}(k) \rho(k x) d \mu(k) .
$$

The last equality follows from the fact (lemma 2.1 ) that $\varphi_{n, C}$ is 0 outside $\bigcup_{i=1}^{n} C^{i}=$ $C^{n},(e \in C$ !).

Using the Lebesgue dominated convergence theorem, it is easy to show that $\theta_{1}$ is continuous. We remark also that $\theta_{1}(x) \neq 0$ implies that there exists $k \in C^{n}$ such that $k x \in U$, in particular $\theta_{1}$ is zero outside $C^{n} U \subset V$. Moreover if $x \in X, k$ and $k^{\prime} \in G$ are such that $\varphi_{n, C}(k) \rho(k x)$ and $\varphi_{n, C}\left(k^{\prime}\right) \rho\left(k^{\prime} x\right)$ are non-zero, we have $k, k^{\prime} \in C^{n}$, $k x, k^{\prime} x \in U$. In particular, we obtain $k k^{\prime-1} \in C^{2 n}$ and $k k^{\prime-1}\left(k^{\prime} x\right) \in U$, hence $k k^{\prime-1} \in C$ by the choice of $U$. Thus we have shown that for each $x \in X$, there exists $k^{\prime} \in G$ such that the function $k \mapsto \varphi_{n, C}(k) \rho(k x)$ is zero outside $C k^{\prime}$. The same property is also true for any function of the form $k \mapsto \varphi_{n, C}\left(k h^{-1}\right) \rho(k x)$ since it is a right translated function obtained from $k \mapsto \varphi_{n, C}(k) \rho[k(h x)]$. Let us now remark that:

$$
\theta_{1}(h x)=\int_{G} \varphi_{n, C}(k) \rho(k h x) d \mu(k)=\int_{G} \varphi_{n, C}\left(k h^{-1}\right) \rho(k x) d \mu(k),
$$

by right invariance of $\mu$. Now using lemma 2.1 , what has just been said above and the right invariance of $\mu$, we obtain that if $h \in C$ and $x \in X$ :

$$
\left|\theta_{1}(h x)-\theta_{1}(x)\right| \leq 2 \mu(C) / n \text {. }
$$


Since $\rho\left(C_{0} F\right)=1$ and $\varphi_{n, C}(C)=1$, we have $\theta_{1}(x) \geq \mu\left(C_{0}\right)$ for each $x \in F$. If we define $\tilde{\theta}_{1}$ by $\left(1 / \mu\left(C_{0}\right)\right) \theta_{1}$, it satisfies properties (ii) and (iii), but it takes values $\geq 1$ and it is only $\geq 1$ on $F$. It is easy to rectify this by composing $\theta_{1}$ with the retraction $r:\left[0, \infty\left[\rightarrow[0,1], r(x)=\inf (x, 1)\right.\right.$. Property (iii) is true for $\theta=r \theta_{1}$ because we have

$$
\left|r(t)-r\left(t^{\prime}\right)\right| \leq\left|t-t^{\prime}\right|, \quad \forall t, t^{\prime} \in[0, \infty[.
$$

Remarks. (1) The function $\theta$ in lemma 2.3 is, in the case where $G=\mathbb{Z}$, very similar to functions constructed in [7, proof of theorem 3] and [8, top of p. 482].

(2) In fact, we need lemma 2.3 in the case where $F$ is compact. Of course, in this context, lemma 2.2 is obvious.

\section{Construction of some skew products}

We consider a continuous action $\alpha$ of the locally compact group $G$ on the normal (Hausdorff) space $X$. We suppose also that our metric space $Y$ satisfies the following condition:

$(\mathscr{H})$ The metric space $Y$ is locally compact, and the group of homeomorphisms with compact support, which are isotopic to the identity through an isotopy with compact support, acts minimally on $Y$.

Another formulation of $(\mathscr{H})$ is the following:

$(\mathscr{H})$ The metric space $Y$ is locally compact, and for every non-void open set $U \subset Y$ and any compact set $K \subset Y$, there exists a finite number of isotopies $\left(k_{i, t}\right)_{t \in[0,1]}$, $i=1, \ldots, n$, with compact support, such that $k_{i, 0}=\mathrm{Id}_{Y}, i=1, \ldots, n$, and $K \subset$ $\bigcup_{i=1}^{n}\left(k_{i, 1}\right)^{-1}(U)$.

Recall that ' $\left(k_{t}\right)_{t \in[0,1]}$ is an isotopy with compact support on $Y$ ' means that the self-map of $Y \times[0,1],(y, t) \mapsto\left(k_{t}(y), t\right)$ is a homeomorphism with compact support. This implies that the set of maps $\left(k_{t}\right)_{t \in[0,1]}$ and $\left(k_{t}^{-1}\right)_{t \in[0,1]}$ are uniformly equicontinuous with respect to any metric on $Y$ compatible with its topology.

We remark that the condition $(\mathscr{H})$ is satisfied if $Y$ is locally compact and given any two points $y, y^{\prime} \in Y$, there exists an isotopy with compact support $\left(k_{t}\right)_{t \in[0,1]}$ such that $k_{1}(y)=y^{\prime}$. This last condition is satisfied in the case where $Y$ is a connected finite dimensional manifold without boundary. It is also satisfied if $Y$ is a connected Hilbert cube manifold (see [3] for the properties of Hilbert cube manifolds).

The condition $(\mathscr{H})$ is also satisfied if $Y$ is a compact space on which a path connected topological group acts minimally.

We now use the results of $\$ 2$ to obtain the following approximation lemma.

Lemma 3.1. Let $\delta>0, C$ a compact subset of $G, K$ and $V$ respectively a compact and an open non-void subset of $Y, U$ an open non-void subset of $X$, be given. We suppose that $F \subset X$ is a closed subset satisfying $g F \cap F=\varnothing$, for all $g \in G, g \neq e$, and $\{g \in G \mid g F \subset U\}$ is not relatively compact in $G$ (in particular it is non-void). Then we can find $H \in \tilde{\mathscr{S}}\left(\mathrm{Id}_{X}\right)$ such that:

(i) $D_{C}\left(\alpha \times \operatorname{Id}_{Y}, H\left(\alpha \times \operatorname{Id}_{Y}\right) H^{-1}\right)<\delta$;

(ii) the set $F \times K$ is contained in the orbit of $U \times V$ under $H\left(\alpha \times \operatorname{Id}_{Y}\right) H^{-1}$.

Proof. Without loss of generality, we can assume that $C$ is a symmetric set containing $e \in G$. We can find a finite number of isotopies of $Y,\left(k_{i, t}\right)_{t \in[0,1]}, i=1, \ldots, n$, with 
compact supports such that $k_{i, 0}=\operatorname{Id}_{Y}$ and $K \subset \bigcup_{i=1}^{i=n}\left(k_{i, 1}\right)^{-1}(V)$. Using the fact that the isotopies have compact support, we can find an $\varepsilon>0$ such that for all $t, t^{\prime} \in[0,1]$, $\left|t-t^{\prime}\right|<\varepsilon$, and for all $i \in\{1, \ldots, n\}$, and all $y \in Y, d\left(k_{i, t}(y), k_{i, t^{\prime}}(y)\right)<\delta$.

Given this $\varepsilon$ and the compact subset $C$ of $G$, we can obtain, using lemma 2.3, a compact subset $C^{\prime}$ of $G$ which will allow us to apply lemma 2.3 .

Since $\{g \in G \mid g F \subset U\}$ is not relatively compact, we can construct by induction $g_{1}, \ldots, g_{n} \in G$ ( $n$ is the same as the number of isotopies) such that

$$
\begin{gathered}
g_{i} F \subset U, \quad i=1, \ldots, n, \\
e \notin C^{\prime} g_{i}, \quad i=1, \ldots, n, \\
C C^{\prime} g_{i} \cap C C^{\prime} g_{j}=\varnothing, \quad 1 \leq i<j \leq n .
\end{gathered}
$$

Applying lemma 2.3 , we can find continuous functions $\varphi_{1}, \ldots, \varphi_{n}: X \rightarrow[0,1]$ such that:

(a) $\varphi_{i}\left(g_{i} F\right)=1$;

(b) $\forall g \in C, \forall x \in X, \forall i \in\{1, \ldots, n\},\left|\varphi_{i}(g x)-\varphi_{i}(x)\right|<\varepsilon$;

(c) $\operatorname{supp} \varphi_{i}$, the (closed) support of $\varphi_{i}$, is so close to $C^{\prime} g_{i} F$ that $\varphi_{i} \mid F=0$, $i=1, \ldots, n$, and $C \operatorname{supp} \varphi_{i} \cap C \operatorname{supp} \varphi_{j}=\varnothing, 1 \leq i<j \leq n$.

We now define $H: X \times Y \rightarrow X \times Y,(x, y) \mapsto\left(x, h_{x}(y)\right)$ by:

$$
h_{x}(y)= \begin{cases}k_{i, \varphi_{i}(x)}(y), & \text { if } x \in \operatorname{supp} \varphi_{i}, \quad i=1, \ldots, n \\ y, & \text { if } x \notin \bigcup_{i=1}^{n} \operatorname{supp} \varphi_{i} .\end{cases}
$$

The condition (c) above shows that $H$ is a well defined homeomorphism. Moreover, this same condition (c) shows that for any $x \in X$ we can find $i \in\{1, \ldots, n\}$ such that for any $g \in C$ (in particular $g=e$ ) and any $y \in Y$,

$$
h_{g x}(y)=k_{i, \varphi_{i}(g x)}(y) \text {. }
$$

It follows then from condition (b) and the definition of $\varepsilon$ that for all $x \in X, g \in C$, and $y \in Y, d\left(h_{g x} h_{x}^{-1}(y), y\right)<\delta$. Since

$$
H\left(\alpha \times \operatorname{Id}_{Y}\right) H^{-1}(g, x, y)=\left(g x, h_{g x} h_{x}^{-1}(y)\right),
$$

we obtain condition (i) of the lemma. The fact that $H$ is in $\tilde{\mathscr{F}}\left(\operatorname{Id}_{X}\right)$ follows easily from the compactness of the supports of the isotopies $\left(k_{i, 1}\right)_{t \in[0,1]}$.

Let us verify condition (ii). Given $y \in K$, we can find $i \in\{1, \ldots, n\}$ such that $k_{i, 1}(y) \in V$. If $x \in F$, we have

$$
H A H^{-1}\left(g_{i}, x, y\right)=\left(g_{i} x, h_{g_{i} x} h_{x}^{-1}(y)\right)
$$

By construction of $g_{i}, g_{i} x \in U$. Since $x \in F$, properties (a) and (c) on the $\left(\varphi_{j}\right)_{j=1, \ldots, n}$ show that $h_{g_{i} x}=k_{i, 1}$ and $h_{x}=\operatorname{Id}_{Y}$, in particular

$$
h_{g_{i} x} h_{x}^{-1}(y)=k_{i, 1}(y) \in V \text {. }
$$

\section{Topologically transitive and minimal skew products.}

In this section, we will suppose that $G$ is a locally compact $\sigma$-compact topological group acting continuously on the normal space $X$. We will suppose that $X$ is second countable (i.e. there exists a countable basis of open sets), and that $Y$ satisfies the condition $(\mathscr{H})$ of $\S 3$. Note that since $Y$ is locally compact and metric, we can 
suppose that the metric $d$ we have on $Y$ is complete. Since $G$ is $\sigma$-compact $\mathscr{P}(\alpha)$ is metrizable and complete, in particular each closed subspace of $\mathscr{S}(\alpha)$ is a Baire space. Since $Y$ satisfies condition $(\mathscr{H})$ of $\S 3$, it is easy to check that the path components of $Y$ are dense, in particular $Y$ is connected. It follows easily from [2, theorem 5, p. 109], that a metric locally compact connected space is $\sigma$-compact, and hence also second countable. We conclude from this that $X \times Y$ is second countable.

Following [6], we introduce $\vartheta(\alpha) \subset \mathscr{P}(\alpha)$, the orbit of $\alpha \times \operatorname{Id}_{Y}$ under the action of the group $\tilde{\mathscr{S}}\left(\mathbf{I d}_{\boldsymbol{X}}\right)$ :

$$
\vartheta(\alpha)=\left\{\boldsymbol{H}\left(\alpha \times \operatorname{Id}_{Y}\right) H^{-1} \mid H \in \tilde{\mathscr{S}}\left(\operatorname{Id}_{X}\right)\right\} .
$$

By what we have seen $\overline{\vartheta(\alpha)}$, the closure of $\vartheta(\alpha)$ in $\mathscr{S}(\alpha)$, is a Baire space.

Let $K$ be compact subset of $X$, such that $g K \cap K=\varnothing$, for all $g \in G, g \neq e$, and for each non-void open subset $U$ of $X$, the set $\{g \mid g K \subset U\}$ is not relatively compact in $G$.

LEMMA 4.1. Under the above hypothesis, there exists a dense $G_{\delta}$ subset $\mathscr{G}$ of the Baire space $\overline{\vartheta(\alpha)}$ such that for each $A$ in $\mathscr{G}$ and each open non-void subset $W$ of $X \times Y$, the orbit of $W$ under $A$ contains the set $K \times Y$.

Proof. Since $\overline{\vartheta(\alpha)}$ is a Baire space, $Y$ is $\sigma$-compact, and $X \times Y$ second countable, it suffices to prove that for each open non-void subset $W$ of $X \times Y$, and each compact subset $\tilde{K}$ of $Y$ the set

$$
b(\tilde{K}, W)=\left\{A \in \overline{\vartheta(\alpha)} \mid K \times \tilde{K} \subset \bigcup_{g \in G} A(g) W\right\}
$$

is open and dense in $\overline{\vartheta(\alpha)}$. We have used $A(g) W$ to denote the image of $W$ under the homeomorphism $(x, y) \mapsto A(g, x, y)$. The fact that $\ell(\tilde{K}, W)$ is open, is an immediate consequence of lemma 1.2. The fact that $\alpha \times \operatorname{Id}_{Y} \in \overline{\mathscr{b}(\tilde{K}, W)}$ results from lemma 3.1. By the definition of $\vartheta(\alpha)$, all we have to show is that $H\left(\alpha \times \operatorname{Id}_{Y}\right) H^{-1}$ is in $\overline{\ell(\tilde{K}, W)}$ for each $H$ in $\tilde{\mathscr{S}}\left(\operatorname{Id}_{X}\right)$. Now we have by lemma 1.3 :

$$
H\left(\alpha \times \operatorname{Id}_{Y}\right) H^{-1} \in \overline{\mathscr{G}(\tilde{K}, W)} \Leftrightarrow \alpha \times \operatorname{Id}_{Y} \in \overline{H^{-1} \mathscr{G}(\tilde{K}, W) H} .
$$

But

$$
\begin{aligned}
H^{-1} G(\tilde{K}, W) H & =\left\{A \in \overline{\vartheta(\alpha)} \mid K \times \tilde{K} \subset \bigcup_{\mathbf{g} \in G} H A H^{-1}(W)\right\} \\
& =\left\{A \in \overline{\vartheta(\alpha)} \mid H^{-1}(K \times \tilde{K}) \subset \bigcup_{g \in G} A(g)\left(H^{-1}(W)\right)\right\} .
\end{aligned}
$$

Since $H$ is a skew product over the identity of $X$, we can find a compact subset $\tilde{\tilde{K}}$ of $Y$ such that $H^{-1}(K \times \tilde{K}) \subset K \times \tilde{K}$. In particular we obtain:

$$
b\left(\tilde{\tilde{K}}, H^{-1}(W)\right) \subset H^{-1} b(\tilde{K}, W) H \text {. }
$$

Since $\alpha \times \operatorname{Id}_{Y} \in \overline{\ell(\tilde{K}, W)}$ holds for any compact set $\tilde{K}$ in $Y$, and any non-void open set $W$ in $X \times Y$, we obtain:

$$
\alpha \times \operatorname{Id}_{Y} \in \overline{\mathscr{b}\left(\tilde{\tilde{K}}, H^{-1}(W)\right)} \subset \overline{H^{-1} \not(\tilde{K}, W) H}
$$

This completes the proof of the lemma. 
We now easily obtain from this lemma two theorems.

TheOREM 4.2. Suppose $Y$ satisfies ( $\mathscr{H}$ ), and that the continuous action $\alpha$ of the $\sigma$-compact locally compact group $G$ on the second countable normal space $X$ is such that there exists a point $x_{0} \in X$ with $G x_{0}$ dense in $X, g x_{0} \neq x_{0}$, for all $g \in G, g \neq e$, and for every compact subset $C$ of $G$ the set $C x_{0}$ has no interior in $X$. Then there exists a skew-product extension $A$ of $\alpha$ to $X \times Y$ which is topologically transitive. In fact, $A$ can be chosen such that every point of the form $\left(x_{0}, y\right) \in X \times Y$ has a dense orbit under the action of $A$.

Proof. Since $G x_{0}$ is dense and for each compact subset $C$ of $G$, the set $C x_{0}$ has no interior in $X$, it is easy to show that $\left\{g \in G \mid g x_{0} \in U\right\}$ is not relatively compact in $G$ for each non-void open subset of $X$. We can now apply lemma 4.1 to find $A \in \mathscr{S}(\alpha)$ such that $\left\{x_{0}\right\} \times Y \subset \bigcup_{g \in G} A(g) W$ for each non-void open set $W$ of $X \times Y$. In particular if $y \in Y$, there exists $g \in G$ such that $\left(x_{0}, y\right) \in A(g) W$, which implies $A\left(g^{-1}\right)\left(x_{0}, y\right) \in W$.

THEOREM 4.3. Suppose there exists a minimal action of a path-connected group on the compact metric space $Y$. Suppose that the locally compact $\sigma$-compact group $G$ acts continuously and minimally on the second countable normal space $X$, in such a way that there exists $x_{0} \in X$, on which the action of $G$ is free, and $C x_{0}$ has no interior in $X$ for each compact subset $C$ of $G$. Then there exists a skew product extension of $G$ to $X \times Y$ which is minimal.

Proof. By theorem 4.2 (or lemma 4.1), we can find a skew product extension $A$ of $\alpha$, such that for every non-void open set $W$ of $X \times Y$, we have $\left\{x_{0}\right\} \times Y$ with $\left\{x_{0}\right\} \times Y \subset \bigcup_{g \in G} A(g) W$. Since $Y$ is compact and $\bigcup_{g \in G} A(g) W$ open, it is easy to find an open neighbourhood $U$ of $x_{0}$ in $X$ such that $U \times Y \subset \bigcup_{g \in G} A(g) W$. The invariance of $\bigcup_{g \in G} A(g) W$ under $G$ implies that

$$
\bigcup_{g \in G} A(g) W \supset\left(\bigcup_{g \in G} g U\right) \times Y=X \times Y,
$$

where the last equality follows from the minimality of the action of $G$ on $X$.

Remarks. (1) In fact in theorems 4.2 and 4.3 (and also in lemma 4.1 if $K \neq \varnothing$ ), the group $G$ has to be second countable (hence metric). This follows from the fact that $G$ is $\sigma$-compact, and the fact that each compact subset $C$ of $G$ is second countable because it is homeomorphic to $C x_{0}$ which is contained in the second countable space $X$.

(2) In the case $X$ compact metric and $G=\mathbb{Z}$, theorem 4.3 is in [6]. In the case $X$ compact, $Y$ a compact connected topological group and $G=\mathbb{Z}$ or $\mathbb{R}$, theorem 4.3 is in [4] and [7].

(3) Theorem 4.2 is well known in the cases $G=\mathbb{Z}$ or $\mathbb{R}$.

(4) J. C. Yoccoz has shown me that theorem 4.3 is false if we do not assume that the metric space $Y$ is compact. In fact, if we look at the action $\alpha$ of $\mathbb{T}^{1}$ on the first factor of $\mathbb{T}^{1} \times \mathbb{R}$, there is no minimal homeomorphism in $\overline{\vartheta(\alpha)}$.

(5) The fact that we must know that $C x_{0}$ has empty interior in $X$, for each compact set in $G$, is necessary for theorems 4.2 and 4.3 . For example if this hypothesis is not 
verified in the context of a minimal action of $G$ on $X$, it is easy to show that the dynamical system $\alpha$ is homeomorphic to the one given by left translation on $G$. However this dynamical system has no minimal extension to $G \times Y$ (as soon as $Y$ has more than one point!), because in a skew product on $G \times X$ the orbits are in fact graphs of (continuous) maps $G \rightarrow Y$.

\section{Existence of minimal actions on Hilbert manifolds}

The purpose of this section is to establish the following theorem.

THEOREM 5.1. Any locally compact, non-compact, second countable topological group acts continuously and minimally on any connected, second countable, infinite dimensional Hilbert manifold.

Fix a topological group $G$ which is locally compact, non-compact and second countable, hence metrizable. We will let $M$ denote a second countable, infinite dimensional Hilbert manifold. We will have to use some results from the theory of infinite dimensional manifolds, most of them can be found either in [1] or in [3]. The first result we will use is the fact that we can find a (connected) Hilbert cube manifold $Y$ such that $M$ is homeomorphic to $l^{2} \times Y$, where $l^{2}$ is the separable infinite dimensional Hilbert space. Then we will try to obtain the minimal action as a skew product of a minimal action on $l^{2}$. Of course by remark 4 at the end of $\S 4$ our methods do not allow us to prove that. What we will prove is that we can find a skew product on $l^{2} \times Y$ whose set of points with a dense orbit is homeomorphic to $l^{2} \times Y$. To prove the existence of such a homeomorphism we will need the notion of $Z$-set, which we recall now.

Definition. (See [1, p. 151]). A closed subset $F$ of a Hilbert manifold $M$ is called a $Z$-subset (or Z-set) if the set of continuous maps $C^{0}(Q, M \backslash F)$ is dense in $C^{0}(Q, M)$ for the uniform topology. We have used $Q$ to denote the product $[0,1]^{\mathbb{N}}$ (i.e. the Hilbert cube).

We have the following theorem $([1$, corollary 7.3, p. 316$])$ :

THEOREM 5.2. If a set $A$, in the Hilbert manifold $M$, is a countable union of (closed) $Z$-sets, then $M \backslash A$ is homeomorphic to $M$.

After these preliminaries of infinite dimensional topology, we now exhibit an action of $G$ on $l^{2}$ with some good properties (see [5]).

We consider the space $C^{0}(G)$ of real valued continuous functions on $G$, endowed with the topology of uniform convergence on compact subsets of $G$. Since $G$ is locally compact and second countable, the space $C^{\circ}(G)$ is in fact a separable Fréchet space. The group $G$ acts continuously on $C^{0}(G)$ by $(g f)(x)=f\left(g^{-1} x\right)$ where $f \in$ $C^{0}(G), g \in G, x \in G$. It is easy to see that $G$ acts effectively on $C^{0}(G)$; this means given any $g \in G, g \neq e$, there exists $f \in C^{0}(G)$ such that $g f \neq f$. By considering functions with compact support, and the fact that $G$ is non-compact, it is easy to show that this action has the properties $(P)$ stated below. 
Definition. A continuous action of $G$ on a topological vector space $E,(g, x) \rightarrow g x$, satisfies properties $(\mathrm{P})$ if:

(i) for each $g \in G$ the map $x \rightarrow g x$ is a (continuous) linear map;

(ii) the vector subspace $\left\{x \in E \mid \lim _{g \rightarrow \infty} g x=0\right\}$ is dense in $E$.

Properties (P) are important because of the following facts.

LEMMA 5.3. If an action of $G$ on $E$ has properties (P), then the action of $G$ on $E^{N}$ also has properties (P).

The proof of lemma 5.3 is easy.

LEMMA 5.4. If an action of $G$ on $E$ has properties (P), and if $E$ is locally convex, then for any compact space $K$ the induced action of $G$ on $C^{0}(K, E)$ (endowed with the uniform or compact open topology) has properties (P).

Proof. Given a convex neighbourhood $V$ of 0 in $E$ and $f: K \rightarrow E$, it is easy to construct a cover $\left(U_{i}\right)_{i=1, \ldots, n}$ of $K$ such that if we choose $k_{i} \in U_{i}, i=1, \ldots, n$, then for all $k \in U_{i}, f(k)-f\left(k_{i}\right) \in V$. Let $\left(\varphi_{i}\right)_{i=1, \ldots, n}$ be a partition of unity on $K$ with supp $\varphi_{i} \subset U$. Using the fact that $V$ is convex, we obtain:

$$
f(k)-\sum_{i=1}^{n} \varphi_{i}(k) f\left(k_{i}\right) \in V, \quad \forall k \in K .
$$

We can, by the density of $\left\{x \in E \mid \lim _{\mathrm{g} \rightarrow \infty} g x=0\right\}$, choose $x_{1}, \ldots, x_{n}$ in this set such that $f\left(k_{i}\right)-x_{i} \in V, i=1, \ldots, n$. Again convexity of $V$ shows that

$$
\sum_{i=1}^{n} \varphi_{i}(k)\left(f\left(k_{i}\right)-x_{i}\right) \in V .
$$

In particular the function defined by

$$
f^{\prime}(k)=\sum_{i=1}^{n} \varphi_{i}(k) x_{i}
$$

is $V+V$ close to $f$. Moreover $\lim _{g \rightarrow \infty} g f^{\prime}=0$, since $f^{\prime}$ takes values in a finite dimensional vector subspace of $\left\{x \mid \lim _{g \rightarrow \infty} g x=0\right\}$ and the action of $G$ is linear.

LEMMA 5.5 (Rolewicz [9]). If the action of $G$ on $E$ has properties (P), then it is topologically transitive.

Proof. Let $U$ and $V$ be open non-void subsets of $E$; we have to show that $g U \cap V$ is non-void for some $g$ in $G$. By properties (P), we can find $a \in U$ and $b \in V$ such that

$$
\lim _{g \rightarrow \infty} g a=\lim _{g \rightarrow \infty} g b=0 .
$$

In particular, if $g$ is big enough, $a+g^{-1} b \in U$ and $g a+b \in V$. But, by the linearity of the action of $g$, we have $g\left(a+g^{-1} b\right)=g a+b$.

Let us go back now to our action of $G$ on $C^{0}(G)$. We will in fact consider the action of $G$ on the separable Fréchet space $X=C^{0}(G)^{\mathrm{N}}$. We have:

LemMA 5.6 (West [10] or [1, p. 168]). The set of continuous maps $F: Q \rightarrow X$ such that for all $g \in G, g \neq e \Rightarrow g f(Q) \cap f(Q)=\varnothing$ is a dense $G_{\delta}$ in $C^{0}(Q, X)$. 
Proof. Since $G$ is second countable and locally compact we can write $G \backslash\{e\}=$ $\bigcup_{n \in \mathbb{N}} C_{n}$ a countable union of compact subsets of $G$. Each of the sets

$$
b_{n}=\left\{f \in C^{0}(Q, X) \mid \forall g \in C_{n}, \quad g f(Q) \cap f(Q)=\varnothing\right\}
$$

is easily seen to be open. This implies that the set defined in the lemma, which is $\bigcap_{n \in N} \ell_{n}$, is a $G_{\delta}$ subset of $C^{0}(Q, X)$. We have still to show the density of this set. Let us use $\left(a_{i}\right)_{i \in \mathbb{N}}$ to denote a dense countable subset of the separable space $C^{0}(G)$. If $f: Q \rightarrow X=C^{0}(G)^{N}$ is a continuous map, we can define for each $m \in \mathbb{N}$ a continuous map $f_{m}: Q \rightarrow C^{0}(G)^{N}$, by

$$
\begin{array}{cc}
p_{n} f_{m}=p_{n} f & \text { if } n<m, \\
p_{n} f_{m}=a_{n-m} & \text { if } n \geq m,
\end{array}
$$

where $p_{n}$ is the $n$th projection of $C^{0}(G)^{\mathrm{N}}$ on $C^{0}(G)$. It is easy to verify that $\lim _{m \rightarrow \infty} f_{m}=f$. Moreover, since $\left(a_{n}\right)_{n \in N}$ is dense in $C^{0}(G)$ and the action of $G$ is effective, given any $g \in G, g \neq e$, there exists $a_{n}$ with $g a_{n} \neq a_{n}$, it follows easily by looking at the $(m+n)$ th component of $f_{m}$ that

$$
g f_{m}(Q) \cap f_{m}(Q)=\varnothing \text {. }
$$

We can now state the properties we need for the action of $G$ on $X$.

Leмма 5.7. There exists a continuous action $\alpha$ of $G$ on a separable Fréchet space $X$, for which there exists a countable dense subset $\left(f_{i}\right)_{i \in N}$ of $C^{0}(Q, X)$ such that:

(a) $\forall i \in \mathbb{N}, \forall g \in G, g \neq e \Rightarrow g f_{i}(Q) \cap f_{i}(Q)=\varnothing$;

(b) $\forall i \in \mathbb{N}, \forall U$ open non-void subset of $X$, the set $\left\{g \in G \mid g f_{i}(Q) \subset U\right\}$ is not relatively compact in $G$.

Proof. We take the action of $G$ on $X=C^{0}(G)^{N}$ given above, we know that (lemma 5.3) it has properties (P). By lemma 5.6, the set $\mathscr{G}$ of functions $f: Q \rightarrow X$ such that for all $g \neq e, g f(Q) \cap f(Q)=\varnothing$ is a dense $G_{\delta}$. Using lemma 5.4 and lemma 5.5 , we obtain that if $U$ is an open non-void subset of $U$, the open subset of $C^{0}(Q, X)$

$$
\mathcal{U}(U)=\left\{f \in C^{\circ}(Q, X) \mid \exists g \in G, \quad g f(Q) \subset U\right\} \text { is dense. }
$$

Since $C^{0}(Q, X)$ is a complete metrizable space, the set $\mathscr{G} \cap \bigcup_{g \in \mathbb{N}} \mathscr{U}\left(U_{j}\right)$ is a dense $G_{\delta}$ subset of $C^{0}(Q, X)$, where $\left(U_{j}\right)_{j \in \mathbb{N}}$ denotes a basis of open non-void subsets of $X$. It is easy to check, using the fact that a compact subset of the infinite dimensional vector space has empty interior, that for every $f \in \bigcap_{i \in N} \mathscr{U}\left(U_{i}\right)$ and every non-void open subset $U$ of $X$, the set $\{g \in G \mid g f(Q) \subset U\}$ is not relatively compact in $G$. Since $C^{0}(Q, X)$ is separable, we can obtain the set $\left(f_{i}\right)_{i \in N}$ by selecting a countable dense subset of $\mathscr{G} \cap \bigcap_{j \in \mathbf{N}} \mathcal{U}\left(U_{j}\right)$.

Proof of theorem 5.1. We will find a skew product action $A$ of $G$ on $X \times Y$ where the action $\alpha$ of $G$ on $X$ is given by lemma 5.7. The fact that $X$ is only a separable Fréchet space and not $l^{2}$ is not a restriction, since all separable infinite dimensional Fréchet spaces are homeomorphic (see [1, theorem 5.2, p. 189]). Remark that, since $Y$ is a connected $Q$-manifold, it satisfies hypothesis $(\mathscr{H})$ via the fact, already mentioned, that isotopies with compact support operate transitively on $Y$. Moreover, if $f_{i}$ is one of the maps $Q \rightarrow X$ given by lemma 5.7, we can apply lemma 4.1 with $f_{i}(Q)$ as a compact subset of $X$. Using the fact that $\overline{\vartheta(\alpha)}$ is a Baire space, we can 
in fact find a $G_{\delta}$ dense subset $\mathscr{M}$ of $\overline{\vartheta(\alpha)}$, such that, for each $A \in \mathscr{M}$, each open non-void subset $U$ of $X \times Y$ and each $i \in \mathbb{N}$, we have:

$$
f_{i}(Q) \times Y \subset \bigcup_{g \in G} A(g) U
$$

It follows easily from the fact that the $\left(f_{i}\right)_{i \in N}$ are dense in $C^{0}(Q, X)$, that for each $A$ in $\mathcal{M}$ and each open non-void subset $U$ of $X \times Y$, the complement in $X \times Y$ of $\bigcup_{g \in G} A(g) U$ is a $Z$-set in $X \times Y$. In particular if $A \in \mathcal{M}$, and $\left(U_{i}\right)_{i \in \mathbb{N}}$ is a basis of open non-void subsets of $X \times Y$, we obtain by theorem 5.2 that $\bigcap_{i \in \mathbb{N}}\left(\bigcup_{g \in G} A(g) U_{i}\right)$ is homeomorphic to $X \times Y$. The action $A$ restricts to a minimal action on $\bigcap_{i \in N}\left(\bigcup_{g \in G} A(g) U_{i}\right)$, since this set is precisely the set of points in $X \times Y$ whose orbit under $A$ is dense in $X \times Y$.

\section{REFERENCES}

[1] C. Bessaga \& A. Pelczynski. Selected Topics in Infinite Dimensional Topology. PWN: Warszawa (1975).

[2] N. Bourbaki. Eléments de Mathématique, livre III, Topologie générale, chapitres 1 \& 2, 4e édition. Herman: Paris (1965).

[3] T. A. Chapman. Lectures on Hilbert Cube Manifolds. CBMS Regional Conference Series in Mathematics, number 28. Amer. Math. Soc: Providence (1976).

[4] R. Ellis. The construction of minimal discrete flows. Amer. J. of Math. 87 (1965), 564-574.

[5] A. Fathi. Existence de systèmes dynamiques minimaux sur l'espace de Hilbert séparable. Topology 22 (1983), 165-167.

[6] S. Glasner \& B. Weiss. On the construction of minimal skew products. Israel J. of Math. 34 (1979), 321-336.

[7] R. Jones \& W. Parry. Compact abelian group extensions of dynamical systems, II. Compositio Math. 25 (1972), 135-147.

[8] J. Mather. Characterization of Anosov diffeomorphisms. Indag. Math. 30 (1968), 479-483.

[9] S. Rolewicz. On orbits of elements. Studia Math. 32 (1969), 17-22.

[10] J. West. Extending certain transformation group actions in separable infinite dimensional Fréchet spaces and the Hilbert cube. Bull. Amer. Math. Soc. 74 (1968), 1015-1019. 